\title{
El Gobierno de la Niñez y la Adolescencia en Situación de Calle. La Producción de Saber y la Cuestión del Sujeto
}

\author{
The Government of the Childhood and the \\ Adolescence in Street Situation. The Production \\ of Knowledge and the Question of the Subject
}
O Governo das crianças e adolescentes em situação de rua. A produção de Saber e a question do sujeito

Diego González García Universidad de la República, Uruguay

Autor referente: dgonzalez@psico.edu.uy

Historia Editorial:

Recibido: 24/03/2017

Aceptado: 20/04/2018

\section{RESUMEN}

El artículo contiene resultados de una investigación realizada en el marco de la maestría en Psicología Social (Facultad de Psicología, Universidad de la República), titulado "El Gobierno de la Niñez y la Adolescencia en Situación de Calle. Un Estudio de la Racionalidad de las Políticas Sociales Focalizadas". El artículo indaga cómo se construyen las nociones de sujeto en las políticas dirigidas a la niñez y la adolescencia en situación de calle. Para ello se presentan los estudios de Gubernamentalidad como cuerpo teórico y metodológico propicio para desarrollar una analítica de las racionalidades políticas y propone el concepto de ciclos de coherencia discursiva para comprender las condiciones de emergencia y procedencias de las producciones de saber vinculados al problema. Desde una metodología cualitativa se analizan documentos oficiales, documentos de difusión y entrevistas a informantes calificados. Los resultados nos aproximan a los modos en que el problema de la niñez y la adolescencia en situación de calle, se han vuelto inteligibles a través de las prácticas 
profesionales, los estudios científicoacadémicos y los discursos

gubernamentales.

Palabras clave: Gubernamentalidad; Racionalidades Políticas; Niñez y Adolescencia; Situación de calle

\section{ABSTRACT}

This paper show the results of a research carried out within the framework of the Master's degree in Social Psychology (Facultad de Psicología, Universidad de la República), titled "The Government of Homeless Childhood and Adolescencent. A Study of the Rationality of Focused Social Policies". The paper analize how the notions of subject are constructed in the politics directed for homeless childhood and adolescence. In order to do so, we present the studies of Governmentality as a theoretical and methodological body propitious to develop an analysis of public policies and propose the concept of discursive coherence cycles to understand the emergency conditions and provenances of knowledge production related to the problem. From a qualitative methodology, official documents, dissemination documents and interviews with qualified informants are analyzed. The results approximate the ways in which the problem of homeless childhood and adolescencent have become intelligible through professional practices, scientific-academic studies and government discourses.

Keywords: Governmentality; Political Racionalities; Childhood and Adolescencent; Homeless

\section{RESUMO}

O artigo contém resultados da pesquisa realizada no âmbito do Mestrado de Psicologia Social (Facultad de Psicología de la Universidad de la República), intitulado "O Governo das Crianças e Adolescentes em situação da rua. Um Estudo da Racionalidade das políticas sociais orientadas". O artigo pesquisa como as noções de sujeito são construídos em as políticas dirigidas as crianças e adolescentes em situação da rua. Se apresenta os estudos de governamentalidade como corpo teórico e metodológico para desenvolver um análise das políticas públicas e se propõe o conceito de

ciclos de coerência discursivo para compreender as condições de emergência e origen das produções de saber ligados ao problema. A partir de uma metodologia qualitativa são analisados documentos oficiais, documentos de transmissão e entrevistas com informantes qualificados. Os resultados nos aproximar ainda mais as formas em que o problema das crianças e adolescentes em situação da rua, se tornaram inteligíveis através das práticas profissionais, os estudos científico-acadêmico e discursos do governo.

Palavras chave: Governamentalidade; Racionalidades Políticas; Crianças e Adolescentes; situação da Rua 


\section{Introducción}

I estudio profundiza sobre las formas de producción de subjetividad vinculadas a los dominios de saber y las técnicas de poder dirigidas a la conducción de la población considerada niños, niñas y adolescentes (NNA) en situación de calle.

El artículo se centra en los dominios de saber, en cómo se construyen las nociones de sujeto en las políticas dirigidas a la niñez y adolescencia en situación de calle. Analiza la producción de discurso en torno a la niñez y la adolescencia en situación de calle en Uruguay desde 1985 a 2015, con la intención de problematizar las nominaciones, clasificaciones y conceptualizaciones que se le ha dado al problema en cuestión. Se presentan los estudios de Gubernamentalidad como cuerpo teórico propicio para desarrollar una analítica de las racionalidades políticas y se propone el concepto de ciclos de coherencia discursiva para comprender las condiciones de emergencia, las relaciones y procedencias de las producciones de saber vinculados a la niñez y la adolescencia en situación de calle.

\section{Estudios de gubernamentalidad y la analítica de las racionalidades políticas}

Los estudios de gubernamentalidad conforman una red de trabajos dedicados al problema de los modos en qué los seres humanos somos gobernados. Estos estudios pretenden analizar la intrincada red que se pone en marcha a través de una serie de tecnologías dirigidas a la conducción de la conducta de una población. De esta manera, se adentran en la descripción de las técnicas y los procedimientos del poder, y en cartografiar los dominios de saber implicados en las prácticas de gobierno. Así, las racionalidades políticas pueden ser entendidas como un conjunto de estrategias dirigidas hacia la conducción de la conducta de la población, en base a unos determinados fines políticos. Las formas de gobierno generan una diversidad de autoridades, como médicos, maestros, educadores, psicólogos, etc; y distintos sujetos de gobierno, como el gobierno de los niños, locos, pobres, etc (Rose, O’Malley, \& 
Valverde, 2012). Foucault (2006) plantea el concepto de gubernamentalidad para comprender la serie de acontecimientos novedosos en relación a la dinámica del poder en el siglo XVIII. Esto refiere a una nueva cuestión social que se desprende del proceso de urbanización de la vida social; la escasez, la circulación y la seguridad.

Entiendo el conjunto constituido por las instituciones, los procedimientos, análisis y reflexiones, los cálculos y las tácticas que permiten ejercer esa forma bien específica, aunque muy compleja, de poder que tiene como blanco principal la población, por forma mayor de saber la economía política, y por instrumento técnico esencial los dispositivos de seguridad (2006, p. 136).

El proceso de "gubernamentalización del Estado" descripto por el autor, tiene como interés el surgimiento del Estado moderno, entendido como la articulación de una serie de tecnologías de conducción de la conducta de los individuos. No debe entenderse que el objetivo de Foucault fuera construir una teoría del Estado, como objeto de estudio o como ente abstracto, sino que el Estado aquí es entendido como efecto de las relaciones de poder y de las prácticas de gobierno que confluyen en la objetivación de los que hoy llamamos Estado

No se puede hablar del Estado cosa como si fuera un ser que se desarrolla a partir de sí mismo y se impone a los individuos en virtud de la mecánica espontanea, casi automática. El Estado es una práctica. No puede disociárselo del conjunto de prácticas que hicieron en concreto que llegara a ser una manera de gobernar, una manera de hacer, una manera, también, de relacionarse con el gobierno (Foucault, 2006, p. 324).

El Estado comporta un arte de gobernar, una manera meditada de administrar y gestionar las riquezas y las poblaciones. Son formas de pensar la práctica de gobierno, de cómo desarrollarlo de la mejor manera y disponiendo los recursos adecuados. Todo arte de gobierno comporta una racionalidad política la cual, ya sea de forma implícita o explícita, se propone dar respuesta a las siguientes preguntas: 
“¿quién o qué debe ser gobernado? ¿Por qué deberían ser gobernados? ¿Cómo deben ser gobernados? ¿Con qué fines deben ser gobernados?” (Rose et al., 2012, p. 116). Esto permite pensar a las formas de gobierno en una diversidad de sujetos de gobierno, de fundamentos, de estrategias y tácticas que se disponen en función de un cálculo de fines. Planteada en base a estas interrogantes, el análisis de la gubernamentalidad implica estudiar las racionalidades políticas de las formas de gobernar determinado problema, en este caso la niñez y la adolescencia en situación de calle. Un estudio de gubernamentalidad implica el análisis de por lo menos tres planos: 1- unas tecnologías de poder, que implican la puesta en marcha de una serie de técnicas dirigidas sobre la conducta de los sujetos; 2- unos saberes específicos, que se producen entorno al objeto que debe ser gobernado; 3- una producción de subjetividad, que se compone por la producción de las poblaciones objetivo del saberpoder y la emergencia de prácticas inmanentes en el proceso dado entre la docilidad y la resistencia de los gobernados. En este artículo nos focalizaremos más en el segundo plano, vinculado a la producción de saberes específicos y especializados que intentan dar respuesta a las interrogantes surgidas del problema de gobierno de los NNA en situación de calle.

\section{Consideraciones metodológicas y perspectiva analítica}

La investigación utilizó una metodología cualitativa (Denzin \& Lincoln, 2003). Las fuentes sistematizadas fueron 11 documentos oficiales (planes, programas, proyectos o evaluaciones que refieren al diseño, planificación y evaluación de las políticas dirigidas NNA en situación de calle), 42 documentos de difusión (sistematizaciones de prácticas, presentaciones en congresos, mesas de discusión, encuentros, entre otros eventos; investigaciones y artículos de revistas vinculadas a la actividad profesional) y 9 entrevistas a informantes calificados (directores, coordinadores y técnicos). La bibliografía sistematizada surge de las entrevistas, páginas webs de organismos 
estatales y ONGs y de bibliotecas especializadas (Instituto Interamericano del Niño y Biblioteca del Centro de Formación de Educación Social). Las fuentes siguen criterios de confiabilidad, pertinencia, vigencia de la información, cobertura geográfica y delimitación cronológica. El período estudiado abarca desde el año1985 hasta el 2015. Todo el material recopilado y analizado se focalizó en el contexto uruguayo.

Los materiales seleccionados y el modo de análisis están vinculados con la perspectiva de los estudios de Gubernamentalidad, los cuales proponen estudiar las racionalidades políticas obviando los textos eruditos de las ciencias políticas o los grandes tratados de filosofía sobre política. En cambio prefieren concentrarse en una literatura menor, producida por pensadores dedicados a tareas de administración, programación y ejecución de las políticas como educadores, psicólogos, trabajadores sociales y otros profesionales (Rose et al., 2012). Este tipo de análisis difiere de una perspectiva del análisis del discurso; donde el lenguaje es entendido en su carácter performativo capaz de moldear la realidad y las subjetividades (Butler, 2002). Los estudios de gubernamentalidad consideran el lenguaje de los textos (planes, programas, proyectos), como un mecanismo entre otros de volver la realidad susceptible de ser gobernada o de establecer la posibilidad de existencia de cierta clase de acciones (Rose et al., 2012). Los textos deben ser comprendidos funcionando dentro de una red de acciones sobre acciones. Los alcances de esta perspectiva se encuentran en la capacidad de dilucidar una racionalidad política, a partir del contenido de los textos. Es posible identificar en los textos una determinada producción de saber y un plan de acción sobre la puesta en marcha de un conjunto de técnicas de conducción de la población.

\section{El saber y la cuestión del sujeto}

Entenderemos el problema del sujeto como una cuestión que compone las formas de gobierno (Rose, 1990). El sujeto es todo aquello a donde se dirigen las acciones para 
conducir su conducta, a través de nominaciones, descripciones, clasificaciones; y también, es todo aquello que se fuga de los dispositivos que lo pretenden gobernar (Deleuze, 1990). Toda práctica de gobierno inventa un sujeto, dispone una serie de técnicas por las cuales conduce su conducta y depende de una producción de conocimiento que lo estudia y lo enuncia. Por lo que, el problema de la niñez y la adolescencia en situación de calle, supone un proceso de construcción de un nuevo dominio de saber-poder, a través de una serie de prácticas dirigidas al abordaje de esta población particular. El sujeto "niño de la calle" emerge en los años 80 en Uruguay, al igual que en toda la región, como un sujeto que vehiculiza una serie de reivindicaciones políticas, apuntadas al respeto de los derechos ciudadanos y a la promoción de las democracias locales. En nombre de este sujeto se apelaba alcanzar una sociedad más justa. Según Fraiman y Rosal (2011), el problema de la niñez y la adolescencia en situación de calle, en Uruguay, se presentaba de forma minoritaria y casi marginal en relación a otros países vecinos como Brasil o Colombia. Los autores sostienen que la categoría de "niños de la calle" se impone en Uruguay, a través de una serie de prácticas sobre una realidad y sobre unos cuerpos que no se corresponden. Si bien es posible corroborar esta idea, a través de los textos de la época, que reflexionan sobre la baja incidencia cuantitativa del problema; no sería pertinente para este artículo revisar la correspondencia entre la "realidad" y la producción de las nominaciones, ya que estas últimas, a nuestro criterio, no funcionan como una mera representación de la realidad, sino como una forma particular de producirla, de configurar una cierta posibilidad de acción. Es más relevante analizar los procesos de descripción, nominación y clasificación que las racionalidades políticas hacen de las poblaciones a las cuales se dirigen. ¿Cuáles son los procedimientos técnicos, que dichas políticas, realizan para producir un saber sobre la población? ¿De qué manera se produce una modificación-sofisticación de estas clasificaciones, para que sean más eficaces como categorías de gobierno? Debemos comprender que la 
potencia de las nominaciones no se encuentra solo en la acción de nombrar o adjudicar características a algo o a alguien, sino que el lenguaje tiene propiedades performativas, entendiéndolo así, "como la práctica reiterativa y referencial mediante la cual el discurso produce los efectos que nombra" (Butler, 2002, p. 18). Sin embargo, este proceso no es solo un acto del lenguaje, sino que las nominaciones y las formas de clasificar las poblaciones conllevan una serie de prácticas, dónde éstas se ejercen y se actualizan en un juego de interacciones. Esta red de prácticas ensambladas son las que efectivamente inventan a los sujetos, los hacen posibles. Lo que sugiere Hacking (2001) es que los procedimientos de clasificación y nominación de categorías, por parte de las ciencias humanas, son literalmente un proceso de "creación de la gente". Afirma que los sujetos se relacionan e interactúan con las clasificaciones a las cuales son asignados y modifican las formas en las que se perciben y se comportan.

Tales clases (de personas y sus conductas) son interactivas. Esta expresión tan fea tiene el mérito de que recuerda nociones como actores, ser agente y acción. El inter puede sugerir la forma en que pueden interactuar la clasificación y el individuo clasificado, la forma en que los actores pueden llegar a conocerse a sí mismos como siendo de una clase, aunque solo sea por ser tratados o institucionalizados como de esa clase, y de este modo tener experiencia de sí mismo en ese sentido (Hacking, 2001, p. 175).

\section{Las formas de saber}

Las formas en que la niñez y la adolescencia en situación de calle en Uruguay, ha sido nominada, estudiada y abordada, se ha modificado desde comienzos de los años 80 . Desde el análisis del contenido de los discursos (Iñiguez, 2006), se desprende que las formas en las que se ha definido y estudiado el problema han sido heterogéneas, apelando a una serie de explicaciones complejas. Estas explicaciones refieren a un campo multicausal donde se implican una serie de factores como: situación de 
"pobreza extrema" de las familias de los NNA, cuestiones socio-económicas particulares que afectan la región, forma acelerada y desordenada de urbanización de la población, problemas de desempleo, "desmembramiento de las familias", ausentismo escolar, fragilidad de algunos barrios para contener a sus integrantes, etc. Del análisis de estas argumentaciones, identificamos tres modos diferenciados de producción de saber sobre el problema. Estos modos, comportan una coherencia específica entre, las formas de estudiar y tratar el problema, y por consiguiente de definir un sujeto al cuál dirigir las acciones gobierno. Los llamaremos ciclos de coherencia discursiva (González, Fagúndez, \& Madjarian, 2009), ya que no necesariamente responden a una cuestión cronológica, sino que conviven y se superponen en un mismo momento histórico; aunque sí representan un proceso de sofisticación en las técnicas de producción de saber sobre la población. Esta noción de ciclo de coherencia discursiva pretende ser fiel con el análisis de las discontinuidades históricas (Foucault, 2009), intenta ser una categoría que dé cuenta de las emergencias discontinuas de los enunciados y de sus procedencias diversas en relación a un conjunto de prácticas particulares. Un ciclo coherente es una serie de enunciados y prácticas que se fundamentan y se sostienen en una misma racionalidad enunciativa. Esto no quiere decir que podamos concebir los textos analizados dentro de un ciclo de coherencia de forma aislada y exentos de sus condiciones políticas e históricas de emergencia, tal como si fuera un ejercicio de clasificación taxonómica de enunciados; más bien significa pensar en racionalidades donde lo relevante son los modos particulares de reflexionar sobre el problema y los argumentos retóricos y técnicos que se evocan para hacerlo inteligible. Por lo que, este ejercicio es únicamente la pretensión de visualizar ciertas coherencias conectivas y las procedencias heterogéneas de los enunciados. 


\section{Ciclo sobre los discursos profesionales}

Estas producciones están concentradas fundamentalmente en los primeros momentos de las prácticas de atención a NNA en situación de calle y se caracterizan por una prolífera producción. Sin embargo, los métodos y las técnicas particulares de este ciclo de coherencia, transversalizan todo el período estudiado. Las producciones se caracterizan por ser sistematizaciones de las prácticas, mesas redondas en encuentros de profesionales, reflexiones en revistas de difusión; donde se encuentran involucrados profesionales de la psicología, trabajo social y de la educación. Estas formas han tomado como referencia empírica los programas de atención de las NNA en situación de calle, tanto los gestionados por organismos estatales como privados (ONGs), para la producción de una metodología y un campo profesional particular. Los textos consultados dan cuenta de las prácticas de atención y relatan las experiencias de la puesta en marcha de un nuevo enfoque, que coloca al niño en el centro de interés de la intervención. Aquí los deseos de los NNA y sus formas particulares de ser, van a ser estudiados y considerados un elemento fundamental para comprender el problema en cuestión. Las técnicas de aproximación al sujeto, requieren ciertas formas de contemplación y comprensión en el terreno particular de la calle, entendida como el "ámbito natural" de vida de los NNA. Por consiguiente, la observación y la interacción casual son los medios de producción de información y de estudio.

Se trata de dedicar mucho tiempo, de no defraudar, de ganar la confianza y la simpatía. Y para ello pasamos dos semanas observándoles en sus lugares naturales, después pasamos al abordaje, lo que en algunos casos significa comprarle las propias cosas que venden para entrar en contacto (Labetkin, 1989, p. 2).

Cual etnógrafos los educadores desarrollan su trabajo de campo en las calles, establecen un vínculo con los NNA, con el objetivo de conocer los aspectos culturales particulares, comprender sus elecciones y voluntades, reconstruir sus historias y llegar 
a los motivos de sus situaciones. Se destacan así, las exhaustivas descripciones de las prácticas que los NNA llevan adelante en la calle, a través de la observación participante y el desarrollo de técnicas lúdicas como medio para explorar y trasmitir conductas, vinculadas a la "escuela, sexualidad, familia, robo, droga, mudanza, entre otros" (Cal \& Zubillaga, 1996, p. 9). Se trata de una serie de procedimientos "experimentales", que pretenden introducirse en el terreno de la calle procurando conceptualizar al sujeto a partir de una constatación empírica.

Este acercamiento es una parte fundamental de la metodología: recorridas, observación, reconocimiento físico, espacios, calles, rincones. No se parte de una planificación de escritorio, sino que de una praxis que permite ir encontrando caminos y conceptualizando. Es necesario un permanente esfuerzo de investigación-acción sobre los chicos y su entorno, la realidad no es estática y hay que ir interpretándola todos los días, es necesario el exhaustivo conocimiento de la calle y su caracterización (Ubilla, 1998, p. 65).

Este conjunto de prácticas delinea un dispositivo de atención-investigación de la niñez y la adolescencia en situación de calle, bajo una serie de procedimientos, que se retroalimentan y se transforman cíclicamente. El orden de ciertos elementos constituye un método, que da cuenta de una racionalidad particular de producción. La sucesión se caracteriza de la siguiente manera: "Contacto directo con los niños, capacitación a través de las situaciones vividas por el equipo de trabajo, evaluación de las actividades y de los resultados obtenidos, adecuar las intervenciones de acuerdo a las evaluaciones realizadas" (Matchin \& Goñez, 2001, p. 249). Estas formas de producción de conocimiento, inmanentes a las prácticas de gobierno (Rose, 1990), despejan un sujeto, constituyéndolo en la población objetivo de atención de los programas calle. La característica más notable de este ciclo, es la intención por definir un sujeto, de hacerlo claramente identificable, intentan responder quiénes son, qué hacen y dónde se encuentran. 
(...) los niños de la calle son niños pobres. Son niños que, usando diferentes estrategias de sobrevivencia, desde un pseudo trabajo -que a veces se parece a la mendicidad- pretenden obtener algo de dinero para sobrevivir. ...Estos niños no van a la escuela y si van, tienen un alto índice de ausentismo, que los conduce inexorablemente a la deserción y en breve tiempo a la peor de las exclusiones sociales. Al acercarnos a las vidas de estos niños observamos que pertenecen a barrios carenciados, con familias que sufren ya varias generaciones de pobreza y necesidades básicas insatisfechas, barrios que por lo general están alejados del centro de la ciudad y de los lugares por donde ellos transitan diariamente. Otros viven hacinados en pensiones o en casas ocupadas en la Ciudad Vieja de Montevideo, o del Centro, formando parte del llamado proceso de tugurización (Álvarez, 2002, p. 22).

Esta caracterización de la población se asemeja en la mayoría de los textos analizados, describe algunas de las posibles prácticas que el sujeto realiza en la calle y agrega coordenadas sobre trayectorias institucionales, procedencias territoriales y de clase. Otras descripciones se concentran en la producción de una imagen, casi fotográfica, de sus comportamientos y apariencia.

Los gurises se muestran agresivos y desconfiados. Por lo general su aspecto físico está deteriorado, pueden pasar varios días sin bañarse, mal dormidos, mal alimentados, estar lastimados, tener hongos, sarna, problemas respiratorios..., es más que un aspecto físico, muchas veces es una pérdida de la auto-estima, una forma de sobrevivencia, una forma de llamar la atención, de gritar su situación con ira, y en ocasiones, con orgullo y rebeldía frente a la sociedad: a veces les gusta dar miedo (Ubilla, 1998, p. 51).

Por último, encontramos caracterizaciones a partir de la enumeración y clasificación de las actividades que desarrollan los NNA, destacando algunas como la mendicidad, prestación de servicios (limpiar parabrisas, hacer malabares, cuidar coches, venta), 
recolección y clasificación de residuos, recreándose y deambulando (Podestá \& Lasida, 2010).

Estas formas de saber, que describen al sujeto en situación de calle, constituyen un cuerpo discursivo conformado por procedimientos metodológicos específicos, que al mismo tiempo en que despliegan un dispositivo asistencial, establecen modos de objetivar sus prácticas para producir conocimiento sobre aquello que intentan gobernar. Así, las prácticas asistenciales tienen un efecto directo de saber sobre los sujetos que gobierna. Se constituye un nuevo "dominio de saber" (Foucault, 2003, p. 12) a partir de las prácticas sociales de asistencia a la niñez y la adolescencia en situación de calle. Se engendran nuevos objetos, conceptos y técnicas que dan lugar a la emergencia de nuevos sujetos. En este sentido, estas producciones buscan consolidarse como una nueva y válida forma de abordaje al problema de la niñez y la adolescencia en situación de calle, con modos particulares de hacer alternativas a las formas disciplinarias de la internación, sustentándose fundamentalmente en la educación no formal y en el discurso basado en los derechos de la niñez y la adolescencia.

\section{Ciclo sobre los discursos científico-académicos}

Las producciones contenidas en ente ciclo de coherencia, son muy dispersas y no representan un volumen significativo, en relación a la producción de saber general del problema. Son un esfuerzo por entender el problema y aportar modelos explicativos. A diferencia de los otros ciclos de coherencia, aquí hay un intento de producir un conocimiento útil basado en una rigurosidad metodológico-científica. Generalmente con un corte cualitativo y proveniente de la psicología, estas investigaciones se concentran en aspectos parciales, aportando mayor profundidad y complejidad a aspectos más generales del problema. La coherencia de estas producciones pasa por la forma que pretenden erigirse como un saber especializado y pertinente sobre el 
fenómeno de los NNA en situación de calle. Los presentamos a continuación priorizando los siguientes aspectos: el problema específico que aborda, la metodología y las conclusiones y sugerencia que se desprende del estudio.

De las producciones más destacables encontramos a Tricotti (1995), la cual desarrolla una investigación que pretende comprender las dinámicas particulares que poseen las familias quienes tienen un integrante en situación de calle. Se interroga por las formas de organización familiar, en relación a su economía, cantidad de integrantes, las formas de vincularse, las modalidades de los roles parentales, nivel educativo, situación laboral, entre otros. Para esto se propone un estudio comparativo entre familias pobres que tienen algún integrante en situación de calle y familias pobres que no tienen integrantes en dicha situación. El estudio de las dinámicas familiares intenta derribar la idea de que la situación de calle está relacionada con la expresión lineal del abandono o de la expulsión familiar, sino con una cierta estructura vincular relacionada con un ejercicio de funciones proveedores y de economía interna de las familias.

Por otro lado, Cura (2002) propone una investigación exploratoria sobre las capacidades "resilientes" de los NNA en situación de calle. Se propone relevar una serie de aptitudes individuales preestablecidas como forma de evaluar las posibilidades de los NNA para superar sus situaciones de riesgo y adversidad e integrarse socialmente. Esta autora comprende, que la capacidad de resiliencia puede desarrollarse en base a la incidencia de ciertos factores protectores, como el sentido del humor, la autoestima, las redes informales, la empatía, entre otros. La constatación de estas aptitudes en la población estudiada le permite concluir que el desarrollo de la resiliencia es una forma satisfactoria de integrarse socialmente.

Ongaro (2002) analiza las respuestas de internación de parte del INAME. A través de un análisis organizacional puede dar cuenta de las trayectorias institucionales de los NNA en situación de calle, entre hogares de amparo, clínicas psiquiatrías y la calle. Finalmente sugiere alternativas "integrales" al problema, con estrategias de restitución 
de derechos, atención a las familias, centros de referencia, familias de acogida y un sistema de información e identificación de la población.

Forselledo (2001) desarrolla la cuestión del uso indebido de drogas en la niñez en situación de calle. Emprende la construcción de un modelo explicativo que incluye variables ambientales, sociales y culturales en la incidencia del consumo de sustancias y coloca a la niñez en situación de calle como una población de riesgo. Finalmente, el autor señala la importancia de crear un modelo basado en la detección de factores de riesgo para desarrollar un modelo preventivo del problema del consumo indebido de drogas.

Por último, Fraiman y Rosal (2011) desarrollan una etnografía en el centro urbano de Montevideo, que pretende entender cómo la población en situación de calle ha llegado a sostener su subsistencia en situación de calle y de qué manera organizan sus vidas cotidianas en este contexto. La investigación profundiza sobre los trayectos institucionales que esta población transita, pasando por un circuito que va desde la calle, la policía, juzgados, proyectos de asistencia, privación de libertad y nuevamente la calle. El estudio ahonda sobre cómo distintas moralidades son puestas en juego, del avance de una moralidad civilizatoria de los derechos de la niñez y la adolescencia, sobre una moralidad de provisión y supervivencia propias de las condiciones de pobreza extrema.

A cerca de estas producciones podemos decir que intentan abordar el problema de forma cualitativa para ahondar en explicaciones sobre cómo es que el problema de la niñez y la adolescencia en situación de calle se produce y con qué elementos está relacionado. En todas las fundamentaciones, las investigaciones pretenden aportar insumos para una mejor comprensión del problema y de esta manera contribuir al diseño de mejores políticas públicas, que incorporen los resultados de estas investigaciones. Resaltamos que las producciones de procedencia académicocientíficas son muy dispersas, en cuanto a los problemas que estudian y las 
metodologías que aplican. Es muy difícil de asegurar un compromiso sistemático con el problema por parte de la academia uruguaya en el período estudiado. Así mismo, esta investigación no se enfocó en el estudio de los impactos que las producciones académicas han tenido sobre las prácticas profesionales y el diseño de las políticas públicas, por lo que no se puede afirmar con certeza cuáles son los aportes de la academia sobre el campo de la niñez y la adolescencia en situación de calle.

\section{Ciclo sobre los discursos gubernamentales}

Estos discursos están vinculados en tres aspectos: 1- su intención es producir diagnósticos nacionales, dimensionamientos cuantitativos, estudios de la población estadísticos y epidemiológicos sobre la niñez y de la adolescencia en situación de calle. 2- los diagnósticos pretenden revelar conocimientos actualizados y confiables de la población para el diseño efectivo de las políticas sociales. 3- entienden que las formas en que fue estudiado el tema han sido escasas, carente de sistematicidad y de índole especulativa. Estos estudios aparecen en el campo de la niñez y la adolescencia en situación de calle, en Uruguay, luego de la crisis económica del 2002 y con la necesidad de elaborar estrategias articuladas y nacionales. El conteo de NNA en situación de calle realizado en el 2003 por ONG Gurises Unidos arrojó un número de 3.100 en Montevideo y el área metropolitana y 4.740 en el interior urbano (Gurises Unidos, 2005). Este estudio monta un precedente en las formas de producción de saber y comprensión de la población. "Por primera vez en el Uruguay se tiene una medición sistemática del número de niños y niñas en situación de calle, utilizando procedimientos científicos validados en la investigación de poblaciones móviles" (Gurises Unidos, 2005, p. 1). Este estudio no solo arroja números, sino que establece un orden de características muy detalladas del grupo estudiado, describiendo prácticas concretas, porcentajes de edades, sexo, distribuciones territoriales, puntos de concentración, trayectos, prevalencia de actividades según las zonas. 
En 2005, se realizaron dos estudios relevantes por parte de entes del Estado. Los mismos pretenden ser diagnósticos abarcativos. El primero, es un estudio epidemiológico sobre el consumo de drogas en la población de NNA en situación de calle, realizado por la Junta Nacional de Drogas (2007). Esta investigación es novedosa, ya que pretende construir una muestra con criterios representativos de la población estudiada.

Comúnmente muchos estudios se han apoyado en posicionamientos especulativos o emocionales, muchas veces fundamentados en casos particulares o de baja representatividad. En este sentido, la disponibilidad de informaciones más amplias y realistas posibilita abarcar el contexto de forma menos tendenciosa, aumentando las probabilidades de lograr políticas más adecuadas a las reales prioridades (Junta Nacional de Drogas, 2007, p. 7).

La muestra de población que se toma en este estudio es comparada con otra población anteriormente estudiada de adolescentes estudiantes de secundaria. Los resultados del estudio están vinculados a prevalencias de consumo, frecuencias, tipos de sustancia (legales e ilegales), relación entre consumo y enfermedad mental, entre consumo y sexualidad, dinámicas de consumo, conformación de la familia de procedencia de los consumidores, entre otros. Se produce información sobre las conductas de los usuarios en relación a los niveles de consumo de sustancias legales e ilegales, las razones de su uso, la percepción del riesgo, de la vulnerabilidad y las expectativas de futuro; con el fin de desarrollar acciones más adecuadas y eficaces.

El segundo estudio, fue encabezado por la Estrategia Nacional para la Atención de NNA en Situación de Calle del Instituto de la Niñez y la Adolescencia del Uruguay (INAU) y el Plan de Investigación y Producción Social del Conocimiento (Infamilia, MIDES). El mismo replica el conteo de NNA en situación de calle realizado por Gurises Unidos en el 2003, solo que éste tomaría únicamente a Montevideo y el área metropolitana. Así, los resultados podían ser comparables y así obtener información 
actualizada de la población estudiada. El resultado de este conteo es de 1887 NNA en situación de calle en la zona de Montevideo y área metropolitana (MIDES, 2007), un 40\% menos que en el 2003. Esta disminución no aparece explicada ni analizada en la bibliografía consultada, por lo que no podemos saber cuáles son los factores que inciden en el aumento o descenso de la población en calle. Más allá de esto, la relevancia de este estudio radica en su capacidad para verter sus resultados en la construcción de las políticas sociales diseñadas posteriormente. Los resultados arrojan, de forma detallada las características de los NNA y consolida distintos niveles de vulnerabilidad y riesgo de las diferentes formas de estar en la calle, que funcionan como un principio taxonómico de la población.

Si bien la construcción de diferentes clases o perfiles de situación de calle, han sido ensayadas desde que los programas calle se han desarrollado (Pérez \& Saravia, 1997; Podestá \& Lasida, 2010), la particularidad de este estudio es que consolida tres perfiles de NNA en situación de calle, los cuales ya se encontraban operando dentro del saber profesional (MIDES, 2007). Estas clasificaciones, aparecen en la bibliografía consultada de forma bastante coincidente planteando tres perfiles, los cuales representan tres niveles de vulneración (Podestá \& Lasida, 2010), dependiendo del proceso de desafiliación o distancia institucional (familia, escuela) que cada situación presenta. Cada grupo presenta diferentes características y formas de estar en la calle. Un primer grupo de "menor grado de vulnerabilidad", el cual se caracteriza por NNA que se localizan en su ámbito barrial, desarrollando actividades de mendicidad y recolección de forma zafral, el período de permanencia en la calle es relativamente escaso. En general, las actividades las realizan en grupo acompañados por adultos, quienes regulan los montos económicos y las horas de trabajo, funcionando como factor de protección, control o explotación. Un segundo grupo de "nivel intermedio de vulnerabilidad", se trata de NNA alejados de sus barrios de referencia, solos o en grupo de pares o acompañados por adultos, transcurren un mayor tiempo en la calle 
desarrollando actividades comerciales, como la venta de estampitas, flores, curitas, etc. Mantiene una relación con sus familias y con su barrio asiduamente, sin embargo, comienzan en un proceso paulatino de alejamiento.

Por último, un grupo de "alto grado vulnerabilidad", también conocido como "perfil crítico" o "calle extrema". Este grupo se encuentra alejado de su familia y su barrio, haciendo de la calle un polo organizador de la vida cotidiana. Han desertado completamente del sistema educativo, generalmente de forma conflictiva. Su itinerario institucional transcurre entre la calle, la policía, el juzgado, INAU en privación de libertad o amparo y nuevamente la calle. Este itinerario que comienza y termina en la calle lo han Ilamado "circuito institucional de violencia" (Fraiman \& Rossal, 2011; Pérez \& Saravia, 1997). Este perfil particular, toma mayor cuerpo en base a los resultados arrojados de dicho estudio (MIDES, 2007), ya que el número de NNA en esta situación de vulnerabilidad extrema ascendía a 111. Por este motivo, se crean una serie de dispositivos especializados para trabajar con esta población hiperfocalizada, que luego constituye los que se llamó "Red de Atención y Protección de Niños/as y Adolescentes en Situación de Calle Extrema INAU-INFAMILIA" (Boffa, 2011). La emergencia de dicho dispositivo responde a la imposibilidad de trabajar con este pequeño grupo NNA el cuál se resistía a las formas tradicionales de atender la situación de calle.

...un grupo bastante identificable de veinte gurises que andaban en la zona Centro, Parque Rodó, Ciudad Vieja, resistente a todo tipo de estrategia de intervención, frente a la búsqueda digamos de esa consolidación de una identidad resistente..., un grupito muy pequeño que eran refractarios ¿no? O sea que ningún tipo de intervención producía un efecto de modificación, y en parte algo de ese discurso de lo refractario estaba presente en esto de la absoluta diferencia del resto ¿no? O sea, estos gurises que son absolutamente diferentes al resto, que ni siquiera los programas Calle que estaban acostumbrados a tratar con población en situación de vulnerabilidad extrema puedan ser abordables (Entrevista a coordinador, 2014) 


\section{Conclusiones}

Hemos visto cómo la producción de saber orientado a la planificación y la gestión de las políticas genera efectos de especialización de las técnicas de abordaje y una sofisticación en las nominaciones, que conlleva la emergencia de nuevas prácticas asistenciales y subjetividades. Hacking (1995) utiliza el término de efecto looping para dar cuenta de las actualizaciones subjetivas constantes producto de la construcción de clases humanas y la interacción de las poblaciones vinculadas con estas últimas. Así, las poblaciones que son clasificadas dentro de ciertas taxonomías, reaccionan organizando sus vidas en torno a dichas clases, ya sea pensándose a través de éstas o por ser vinculados con las mismas. Las clasificaciones performan a los individuos y estás transformaciones subjetivas funcionan, a su vez, modificando el campo empírico al cual dichas clasificaciones hacen referencia, por lo tanto, estas deben ser reformuladas para dar cuenta de la novedad. En estos términos, la niñez y la adolescencia en situación de calle es el proceso por el cual ha sido nombrada, estudiada y abordada. Un proceso formado históricamente, que ha tenido la capacidad técnica de articular una serie de prácticas y de procedimientos para definir y construir un nuevo sujeto dentro del campo de la infancia. Un sujeto que se ha vuelto inteligible por una heterogeneidad de racionalidades enunciativas compuestas por prácticas profesionales, estudios científico-académicos y discursos gubernamentales. Hemos reflexionado cómo la construcción de poblaciones focalizadas implica la construcción de nuevos instrumentos técnicos para medir, clasificar y localizar. Estas formas dan lugar a la emergencia de nuevas categorías subjetivas y nuevos dispositivos de atención inmanentes a las prácticas de gobierno. 


\section{Referencias}

Álvarez, C. (2002). Una mirada desde la Educación Social al problema de los niños en situación de calle. Revista Nosotros. Publicación técnica del Instituto Nacional del Menor, 1(11-12), 20-25.

Boffa, J. (2011). Red de Atención y Protección de Niños/as y Adolescentes -INAUINFAMILIA. Recuperado de https://apudialogosocial.wordpress.com/2011/09/21/red-de-atencion-yproteccion-de-ninosas-y-adolescentes/

Butler, J. (2002). Cuerpos que importan. Sobre los límites materiales y discursivos del «sexo». Barcelona: Paidós.

Cal, M., \& Zubillaga, B. (1996). Niños en la calle: un abordaje en situación. Revista Nosotros. Publicación técnica del Instituto Nacional del Menor, 1(4), 7-11.

Cura, V. (2002). Capacidades en niños y adolescentes para la superación del riesgo de las situaciones de calle y el logro de la integración social. En Políticas de niñez y adolescencia. Aportes y estrategias para la acción (p. 121-143). Montevideo: Universidad Católica- INAME.

Deleuze, G. (1990). ¿Qué es un dispositivo? En Michel Foucault, filósofo (p. 155-163). Barcelona: Gedisa.

Denzin, N. K., \& Lincoln, Y. S. (Eds.). (2003). The landscape of qualitative research: theories and issues. Thousand Oaks, Calif.: Sage Publications.

Forselledo, A. (2001). Niñez en situación de calle. Un modelo de prevención de las farmacodependencias basado en los derechos humanos. Boletín del Instituto Interamericano del Niño, $\mathrm{N}^{\circ} 236,49-80$.

Foucault, M. (2003). La verdad y las formas jurídicas. Barcelona: Gedisa.

Foucault, M. (2006). Seguridad, territorio, población: curso en el Collége de France (1977-1978). Buenos Aires: FCE; 
Foucault, M. (2009). La Arqueología Del Saber. (Siglo XXI de España, Ed.). Madrid: Siglo XXI.

Fraiman, R., \& Rossal, M. (2011). De calles, trancas y botones: una etnografía sobre violencia, solidaridad y pobreza urbana. Montevideo, Uruguay: División Programas y Proyectos de la Subsecretaría del Ministerio del Interior.

González, D., Fagúndez, D., \& Madjarian, M. (2009). Investigación sobre las producciones histórico-discursivas acerca problemática de niños, niñas y adolescentes en situación de calle en Uruguay entre los años 1985 y 2009. Recuperado de http://www.estudiantes.csic.edu.uy/2015/09/02/fpsiinvestigacion-sobre-las-producciones-historico-discursivas-acercaproblematica-de-ninos-ninas-y-adolescentes-en-situacion-de-calle-en-uruguayentre-los-anos-1985-y-2009/

Gurises Unidos. (2005). Niños, niñas y adolescentes en situación de calle en Uruguay ¿Cuántos son? Recuperado de http://www.gurisesunidos.org.uy/

Hacking, I. (1995). The looping effects of human kinds. En D. Sperber, D. Premack, \& J. Premack (Eds.), Causal cognition: a multi-disciplinary debate (Oxford Univ. Press). Oxford.

Hacking, I. (2001). ¿La construcción social de qué? Barcelona: Paidós.

Iñiguez, L. (2006). Análisis del discurso. Catalunya: Editorial UOC.

Junta Nacional de Drogas. (2007). Niños, niñas y adolescentes en situación de calle: un estudio descriptivo-exploratorio sobre consumo de drogas y factores asociados (Investigación). Montevideo: Junta Nacional de Drogas.

Labetkin, M. (1989). Uruguay: El reto de reinsertar a los niños marginados. Fundación para la protección de la infancia dañada por los estados de emergencia, 1-2.

Matchin, M., \& Goñez, P. (2001). Proyecto centro abierto. Atención de niños en situación de calle y su entorno familiar. En 40 Encuentro Nacional de Educadores: las prácticas educativo sociales con niños, niñas y adolescentes 
en la vida cotidiana: Montevideo, 11, 12 y 13 de setiembre de 2000, Sala Azul de la Intendencia Municipal de Montevideo (p. 243-253). Montevideo, Uruguay: Oficina de UNICEF en Uruguay: Centro de Formación y Estudios del INAME. MIDES. (2007). Estudio de Dimensionamiento de la Situación de calle de niños, niñas y adolescentes. Montevideo, Uruguay.

Ongaro, A. M. (2002). El internado, ¿es una respuesta válida en situación de calle? Su diagnóstico. Su análisis. Propuestas alternativas. En Políticas de niñez y adolescencia. Aportes y estrategias para la acción (p. 85-99). Montevideo: Universidad Católica- INAME.

Pérez, D., \& Saravia, C. (1997). El trabajo del educador en las diferentes áreas de abordaje. Revista Nosotros. Publicación técnica del Instituto Nacional del Menor, 1(6), 32-34.

Podestá, M., \& Lasida, J. (2010). Herramientas para el abordaje integral de niños, niñas y adolescentes en situación de calle. Montevideo: Gurises Unidos Telefónica.

Rose, N. (1990). Governing the soul: the shaping of the private self. London; New York: Routledge.

Rose, N., O’Malley, P., \& Valverde, M. (2012). Gubernamentalidad. Astrolabio, O(8). Recuperado de http://revistas.unc.edu.ar/index.php/astrolabio/article/view/2042

Tricotti, L. (1995). La situación de calle: ¿abandono o solidaridad familiar? [Montevideo]: CLAEH: Gurises Unidos.

Ubilla, P. (1998). El ómnibus de El Abrojo.Un recorrido con I@s guris@s en situación de calle. Montevideo: Multiversidad Franciscana de América Latina. El Abrojo. 
Formato de citación

González, D. (2018). El Gobierno de la Niñez y la Adolescencia en Situación de Calle.

La Producción de Saber y la Cuestión del Sujeto. Psicología, Conocimiento y Sociedad, 8(1), 238-261. doi: http://dx.doi.org/10.26864/PCS.v8.n1.11 